\title{
Precise QCD predictions for the production of a photon pair in association with two jets
}

\author{
Gehrmann, T ; Greiner, N ; Heinrich, G
}

\begin{abstract}
We compute the cross section for the production of a high-mass photon pair in association with two hadronic jets to next-to-leading order in quantum chromodynamics. Our results allow us for the first time to reliably predict the absolute normalization of this process and demonstrate that the shape of important kinematical distributions is modified by higher-order effects. The perturbative corrections will be an important ingredient in precision studies of Higgs boson properties from its production in association with two jets.
\end{abstract}

DOI: https://doi.org/10.1103/PhysRevLett.111.222002

Posted at the Zurich Open Repository and Archive, University of Zurich

ZORA URL: https://doi.org/10.5167/uzh-89904

Journal Article

Accepted Version

Originally published at:

Gehrmann, T; Greiner, N; Heinrich, G (2013). Precise QCD predictions for the production of a photon pair in association with two jets. Physical Review Letters, 111(22):online.

DOI: https://doi.org/10.1103/PhysRevLett.111.222002 


\title{
Precise QCD predictions for the production of a photon pair in association with two jets
}

\author{
T. Gehrmann ${ }^{a}$, N. Greiner ${ }^{b}$, G. Heinrich ${ }^{b}$ \\ a Institute for Theoretical Physics, University of Zürich, CH-8057 Zürich, Switzerland \\ ${ }^{b}$ Max Planck Institut für Physik, Föhringer Ring 6, D-80805 München, Germany
}

\begin{abstract}
We compute the cross section for the production of a high-mass photon pair in association with two hadronic jets to next-to-leading order (NLO) in quantum chromodynamics (QCD). Our results allow for the first time to reliably predict the absolute normalisation of this process, and demonstrate that the shape of important kinematical distributions is modified by higher-order effects. The perturbative corrections will be an important ingredient to precision studies of Higgs boson properties from its production in association with two jets.
\end{abstract}

PACS numbers: $12.38 \mathrm{Bx}$

Following the discovery of the Higgs boson at the CERN LHC [1], the study of Higgs boson properties has now become a major research objective of particle physics. By measuring a variety of production and decay modes of the Higgs boson, the determination of the Higgs boson quantum numbers and couplings to Standard Model particles will become increasingly precise, thereby allowing to uncover possible deviations from the Standard Model realization of the Higgs mechanism of electroweak symmetry breaking. These studies rely on a close interplay between experimental data and theoretical predictions for Higgs boson signal and background processes.

Reliable theoretical predictions for hadron collider processes require the inclusion of higher order QCD corrections. Impressive progress has been made in recent years in the derivation of QCD corrections to the most important Higgs boson production processes, with gluon fusion [2] and associated production [3] known fully exclusively to next-to-next-to-leading order (NNLO) in QCD, and vector-boson-fusion [4] and associated production with top quarks [5] to next-to-leading order (NLO). For the gluon fusion process, which is the largest contribution to Higgs production at the LHC, NLO corrections have also been derived for Higgs boson production in association with up to three jets [6-8].

To disentangle different Higgs boson production processes, and to optimize signal-to-background ratios, one often distinguishes samples according to the number of jets observed together with the Higgs boson candidate. In particular, Higgs production in association with two jets allows to probe the vector boson fusion process, which is of crucial importance for the study of the Higgs mechanism and for the determination of couplings. Accordingly, precise predictions for the corresponding background processes are required to optimize detection strategies and to allow for a meaningful interpretation of experimental observations.

Among the most prominent Higgs boson discovery modes is the decay to two photons $H \rightarrow \gamma \gamma$ [11], which, despite its low branching fraction, has a favourable signal-to-background ratio. In the experimental studies of Higgs boson properties in this channel [9, 10], the background contributions are estimated from fits to side-band data with diphoton invariant mass away from the Higgs boson mass. This pragmatic approach to quantify the total background allowed the Higgs boson discovery in the diphoton channel; it may however face its limitations once it comes to precision studies of the Higgs boson properties, in particular its production mechanism. In this context, it is highly desirable to have precise predictions for the production of photon pairs in association with a definite number of hadronic jets. Photon pair production without extra jets is known to NNLO [12], and photon-pair-plus-one-jet production to NLO $[13,14]$. In both cases, the inclusion of higher order corrections revealed new kinematical features that may turn out to be crucial in precision studies. For photon-pair-plus-two-jet production, only leading order predictions were available up to now, which are insufficient for precise phenomenological studies. In this letter, we present the first calculation of NLO QCD corrections to this process.

Photons at hadron colliders can originate either from the hard interaction process itself or from hadron decays. To single out the photons originating from the hard production process, photon isolation criteria are applied, which are typically formulated in the form of a maximum amount of hadronic energy that is admitted in the vicinity of the photon. By admitting some hadronic activity around a photon, one includes final state configurations with a final state quark radiating a highly energetic collinear photon. These configurations contain a collinear singularity, related to small invariant masses of the quark-photon system. Mass factorization in QCD relates this singularity to a redefinition of the quark-tophoton fragmentation function $[15,16]$, which describes the production of a photon inside a hadronic jet. Like parton distributions in the proton, these fragmentation functions are non-perturbative objects that have to be determined from experimental data [17].

To suppress the dependence of isolated photon cross sections on these a priori unknown fragmentation func- 
tions, a smooth cone isolation criterion has been proposed [18], which varies the threshold on the hadronic energy inside the isolation cone with the radial distance from the photon. It is described by the cone size $R$, a weight factor $n$ and an isolation parameter $\epsilon$. With this criterion, one considers smaller cones of radius $r_{\gamma}$ inside the $R$-cone and calls the photon isolated if the energy in any sub-cone does not exceed

$$
E_{\text {had,max }}\left(r_{\gamma}\right)=\epsilon p_{T}^{\gamma}\left(\frac{1-\cos r_{\gamma}}{1-\cos R}\right)^{n} .
$$

By construction, the smooth cone isolation does not admit any hard collinear quark-photon configurations, thereby allowing a full separation of direct and secondary photon production, and consequently eliminating the need for a photon fragmentation contribution in the theoretical description. We will employ the smooth isolation criterion throughout our calculation.

For the production of two photons and two jets the calculation of three types of subprocesses is needed, namely

$$
q \bar{q} \rightarrow \gamma \gamma q \bar{q}, \quad q \bar{q} \rightarrow \gamma \gamma q^{\prime} \bar{q}^{\prime}, \quad g g \rightarrow \gamma \gamma q \bar{q} .
$$

All other subprocesses can be obtained by crossing and/or changing of overall prefactors. We neglect the contributions from the loop-suppressed process $g g \rightarrow$ $\gamma \gamma g g$, which is formally of higher order in the perturbative expansion. To obtain the NLO predictions for each subprocess, virtual one-loop corrections and single real radiation corrections have to be computed.

The matrix elements for tree level and real emission contributions have been generated with MadGraph [19], the subtraction terms to cancel the QCD singularities are provided by MadDipole [20], which uses the dipole formalism as described in [21]. The tree level and IR subtracted NLO real radiation cross sections for diphoton plus two jet final states have been checked against SHERPA [22], finding good agreement. For the generation of the virtual one-loop amplitudes the package GoSam [23] has been used. Based on a Feynman diagrammatic approach, it uses QGRAF [24] and FORM [25] to generate the diagrams. Furthermore it uses the library Spinney [26] to deal with the spinor-helicity formalism and Haggies [27] and FORM to optimise the output. For the reduction we use a $d$-dimensional integrand level decomposition as implemented in Samurai [28], applying unitarity based methods [29]. For unstable points we used a tensorial decomposition as contained in Golem95 [30]. The remaining master integrals are computed with either OneLoop [31], or Golem95C [30]. Contributions from top quarks loops are omitted as they have been shown to be negligible in the diphoton plus one jet process [14]. All ingredients are combined in an automated way with a numerical phase space integration provided by MadEvent [32].

The numerical results presented in the following have been calculated at center-of-mass energy $\sqrt{s}=8 \mathrm{TeV}$.
For the jet clustering we used an anti- $k_{T}$ algorithm [33] with a cone size of $R_{j}=0.5$ provided by the FastJet package [34]. We used the CT10 set of parton distributions [35] as contained in the LHAPDF library [36] and worked with $N_{F}=5$ massless quark flavours. The following kinematic cuts have been applied:

$$
\begin{aligned}
& p_{T}^{\text {jet }}>30 \mathrm{GeV}, \quad p_{T}^{\gamma, 1}>40 \mathrm{GeV}, \quad p_{T}^{\gamma, 2}>25 \mathrm{GeV} \\
& \left|\eta^{\gamma}\right| \leq 2.5, \quad\left|\eta^{j}\right| \leq 4.7, \quad R_{\gamma, j}>0.5, \quad R_{\gamma, \gamma}>0.45
\end{aligned}
$$

For the photon isolation, we use the smooth cone isolation criterion [18] with $R=0.4, n=1$ and $\epsilon=0.05$. Renormalization and factorization scales $\mu$ and $\mu_{F}$ have been chosen as dynamical scales, with the default scale being $\mu_{0}^{2}=\frac{1}{4}\left(m_{\gamma \gamma}^{2}+\sum_{j} p_{T, j}^{2}\right)$, and we have used $\mu=\mu_{F}$. The behavior of the total cross section when varying the scales by a factor of $x \cdot \mu_{0}$ is shown in Figure 1 . The plot shows a substantial reduction of the scale uncertainty when including the NLO corrections. For the total cross section we obtain

$$
\sigma_{\mathrm{LO}}=2.39_{-0.49}^{+0.66} \mathrm{pb}, \sigma_{\mathrm{NLO}}=3.08_{-0.18}^{+0.21} \mathrm{pb},
$$

where the perturbative uncertainty is estimated by varying $x \in[0.5,2]$.

In Higgs production via vector boson fusion (VBF) and Higgs decay into two photons, the CP-properties of the Higgs coupling to electroweak gauge bosons are reflected in the azimuthal distribution of the two tagging jets [37]. Fig. 2 shows the distribution of the corresponding QCD background for the production of two jets and two photons. The error band denotes the theoretical uncertainty

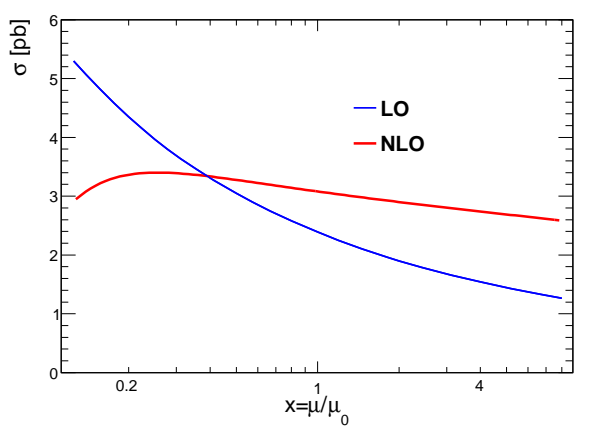

FIG. 1. Scale dependence of the total cross section at $\mathrm{LO}$ and NLO with $x=\mu / \mu_{0}$.

estimated from the variation of renormalization and factorization scales from $0.5 \cdot \mu_{0}$ to $2 \cdot \mu_{0}$. Apart from the expected reduction of the scale uncertainty, Figure 2 exhibits a significant change of the NLO shape compared to the tree-level calculation. This behavior can be understood from the fact that the additional parton present in the NLO real radiation part enhances the configurations where the jets are close in azimuthal angle. Therefore 


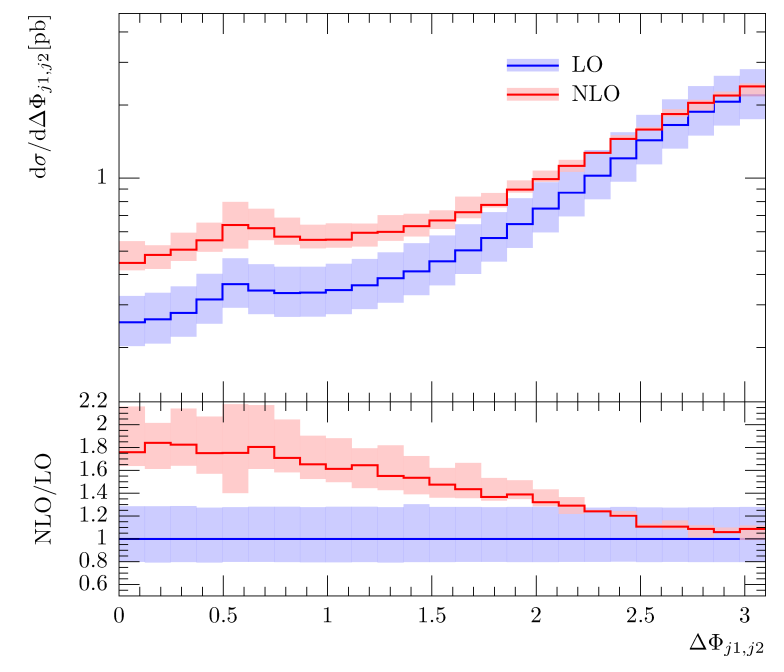

FIG. 2. Azimuthal angle $\Delta \Phi\left(j_{1}, j_{2}\right)$ distribution between the two hardest jets.

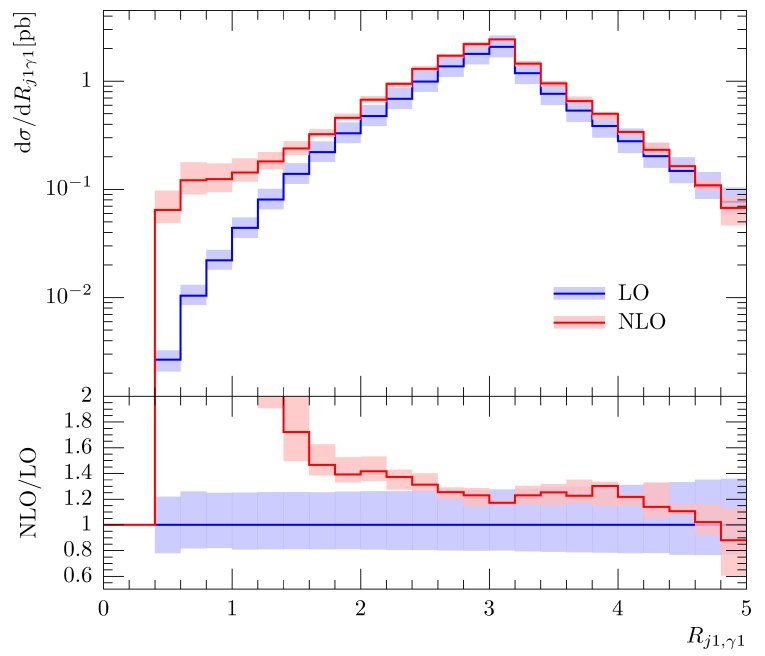

FIG. 3. $R$-separation $R\left(j_{1}, \gamma_{1}\right)$ between the hardest jet and the hardest photon.

it is crucial that NLO corrections are taken into account for a precise estimation of the background.

The $R$-separation between the hardest jet and the hardest photon as shown in Figure 3 exhibits large differences in the shape at NLO for small values of the $R$-separation. This can be explained by the underlying kinematics. At LO, the pair of hard photon and hard jet being close in $R$-space would have to be counterbalanced with the soft photon and the soft jet. This is kinematically impossible at LO but appears at NLO due to the additional radiation.

Figure 4 shows the invariant mass of the diphoton system. Here as well, inclusion of the NLO corrections results in a kinematics-dependent, non-constant correction factor. In particular, NLO corrections lead to an enhancement in the low $m_{\gamma \gamma}$ regime. This behavior can be

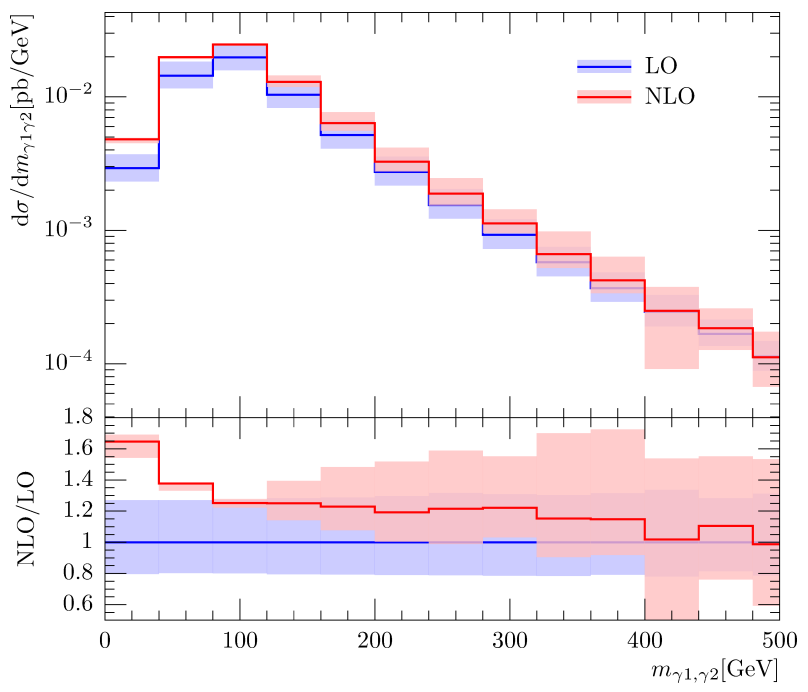

FIG. 4. Invariant mass $m_{\gamma \gamma}$ of the two photons.

explained by the larger final-state phase space available at NLO. The increase of the perturbative uncertainty in the high-mass tail can be understood to be due to the growing relative importance of three-jet final states in this region, thereby resulting in a leading order scale dependence.

The substantial corrections to the shapes that we observed in several different kinematic distributions are well beyond the estimated uncertainties obtained at leading order. They highlight the importance of genuine NLO QCD effects in photon-pair-plus-two-jet production. To obtain background estimates in Higgs boson studies from the candidate-plus-two-jet events sample, multi-differential distributions are required. Those can often not be extracted reliably from sideband data due to lack of statistics, and our calculation provides for the first time a sound theoretical prediction for the QCD induced background in the highly important two-photon channel. With the cuts as defined above, we observe that the total cross section can be predicted at NLO to an accuracy of about 10\%. At present, our calculation is restricted to the smooth cone isolation, which eliminates photon-fragmentation contributions and therefore differs from the fixed-cone isolation prescription typically used in experimental studies. A more detailed phenomenological study of higher-order QCD effects in diphoton-plustwo-jet production and of the impact of photon isolation issues will be presented in a subsequent paper.

We thank the other members of the GoSam collaboration for useful discussions. N.G. and G.H. want to thank Thomas Hahn for technical support of the computing resources, Gionata Luisoni for his help with Rivet and Sherpa, and the University of Zurich for kind hospitality while parts of this project were carried out. This work was supported in part by the Schweizer National- 
fonds under grant 200020-138206, and by the Research Executive Agency (REA) of the European Union under the Grant Agreement number PITN-GA-2010-264564 (LHCPhenoNet). We acknowledge use of the computing resources of the Rechenzentrum Garching.

[1] G. Aad et al. [ATLAS Collaboration], Phys. Lett. B 716 (2012) 1 [arXiv:1207.7214]; S. Chatrchyan et al. [CMS Collaboration], Phys. Lett. B 716 (2012) 30 [arXiv:1207.7235].

[2] C. Anastasiou, K. Melnikov and F. Petriello, Nucl. Phys. B 724 (2005) 197 [hep-ph/0501130]; M. Grazzini, JHEP 0802 (2008) 043 [arXiv:0801.3232].

[3] G. Ferrera, M. Grazzini and F. Tramontano, Phys. Rev. Lett. 107 (2011) 152003 [arXiv:1107.1164].

[4] T. Figy, C. Oleari and D. Zeppenfeld, Phys. Rev. D 68 (2003) 073005 [hep-ph/0306109]; K. Arnold, et al., Comput. Phys. Commun. 180 (2009) 1661 [arXiv:0811.4559]; F. Campanario, T. M. Figy, S. Plätzer and M. Sjödahl, arXiv:1308.2932.

[5] W. Beenakker, S. Dittmaier, M. Kramer, B. Plumper, M. Spira and P. M. Zerwas, Nucl. Phys. B 653 (2003) 151 [hep-ph/0211352]; S. Dawson, C. Jackson, L. H. Orr, L. Reina and D. Wackeroth, Phys. Rev. D 68 (2003) 034022 [hep-ph/0305087]; R. Frederix, S. Frixione, V. Hirschi, F. Maltoni, R. Pittau and P. Torrielli, Phys. Lett. B 701 (2011) 427 [arXiv:1104.5613].

[6] D. de Florian, M. Grazzini, Z. Kunszt, Phys. Rev. Lett. 82 (1999) 5209 [hep-ph/9902483]; V. Ravindran, J. Smith, W. L. Van Neerven, Nucl. Phys. B634 (2002) 247 [hep-ph/0201114].

[7] J. M. Campbell, R. K. Ellis, G. Zanderighi, JHEP 0610, 028 (2006). [hep-ph/0608194];

J. M. Campbell, R. K. Ellis, C. Williams, Phys. Rev. D81 (2010) 074023. [arXiv:1001.4495]. H. van Deurzen, N. Greiner, G. Luisoni, P. Mastrolia, E. Mirabella, G. Ossola, T. Peraro and J. F. von Soden-Fraunhofen et al., Phys. Lett. B 721, 74 (2013) [arXiv:1301.0493].

[8] G. Cullen, H. van Deurzen, N. Greiner, G. Luisoni, P. Mastrolia, E. Mirabella, G. Ossola, T. Peraro and F. Tramontano, arXiv:1307.4737.

[9] [ATLAS Collaboration], ATLAS-CONF-2013-012.

[10] [CMS Collaboration], CMS-PAS-HIG-13-001.

[11] J. R. Ellis, M. K. Gaillard and D. V. Nanopoulos, Nucl. Phys. B 106 (1976) 292; M. A. Shifman, A. I. Vainshtein, M. B. Voloshin and V. I. Zakharov, Sov. J. Nucl. Phys. 30 (1979) 711.

[12] S. Catani, L. Cieri, D. de Florian, G. Ferrera and M. Grazzini, Phys. Rev. Lett. 108 (2012) 072001 [arXiv:1110.2375].

[13] V. Del Duca, F. Maltoni, Z. Nagy and Z. Trocsanyi, JHEP 0304 (2003) 059 [hep-ph/0303012].

[14] T. Gehrmann, N. Greiner and G. Heinrich, JHEP 1306 (2013) 058 [arXiv:1303.0824].

[15] K. Koller, T. F. Walsh and P. M. Zerwas, Z. Phys. C 2 (1979) 197.

[16] E. W. N. Glover and A. G. Morgan, Z. Phys. C 62 (1994) 311; A. Gehrmann-De Ridder and E. W. N. Glover, Nucl.
Phys. B 517 (1998) 269 [hep-ph/9707224].

[17] D. Buskulic et al. [ALEPH Collaboration], Z. Phys. C 69 (1996) 365; A. Gehrmann-De Ridder, T. Gehrmann and E.W.N. Glover, Phys. Lett. B 414 (1997) 354 [hep-ph/9705305].

[18] S. Frixione, Phys. Lett. B 429 (1998) 369 [hep-ph/9801442].

[19] T. Stelzer and W. F. Long, Comput. Phys. Commun. 81, 357 (1994) [hep-ph/9401258]; J. Alwall et al., JHEP 0709, 028 (2007) [arXiv:0706.2334].

[20] R. Frederix, T. Gehrmann and N. Greiner, JHEP 0809, 122 (2008) [arXiv:0808.2128]; JHEP 1006, 086 (2010) [arXiv:1004.2905].

[21] S. Catani and M. H. Seymour, Nucl. Phys. B 485, 291 (1997) [Erratum-ibid. B 510, 503 (1998)] [hep-ph/9605323].

[22] T. Gleisberg, S. Hoeche, F. Krauss, M. Schonherr, S. Schumann, F. Siegert and J. Winter, JHEP 0902, 007 (2009) [arXiv:0811.4622].

[23] G. Cullen, N. Greiner, G. Heinrich, G. Luisoni, P. Mastrolia, G. Ossola, T. Reiter and F. Tramontano, Eur. Phys. J. C 72, 1889 (2012) [arXiv:1111.2034].

[24] P. Nogueira, J. Comput. Phys. 105, 279 (1993).

[25] J.A.M. Vermaseren, math-ph/0010025. J. Kuipers, T. Ueda, J.A.M. Vermaseren and J. Vollinga, Comput. Phys. Commun. 184, 1453 (2013) [arXiv:1203.6543].

[26] G. Cullen, M. Koch-Janusz and T. Reiter, Comput. Phys. Commun. 182, 2368 (2011) [arXiv:1008.0803].

[27] T. Reiter, Comput. Phys. Commun. 181, 1301 (2010) [arXiv:0907.3714].

[28] P. Mastrolia, G. Ossola, T. Reiter and F. Tramontano, JHEP 1008, 080 (2010) [arXiv:1006.0710].

[29] R. K. Ellis, W.T. Giele and Z. Kunszt, JHEP 0803, 003 (2008) [arXiv:0708.2398]. G. Ossola, C.G. Papadopoulos and R. Pittau, Nucl. Phys. B 763, 147 (2007) [hep-ph/0609007]. P. Mastrolia, G. Ossola, C.G. Papadopoulos and R. Pittau, JHEP 0806, 030 (2008) [arXiv:0803.3964]. G. Ossola, C.G. Papadopoulos and R. Pittau, JHEP 0805, 004 (2008) [arXiv:0802.1876]. G. Heinrich, G. Ossola, T. Reiter and F. Tramontano, JHEP 1010, 105 (2010) [arXiv:1008.2441].

[30] T. Binoth, J.P. Guillet, G. Heinrich, E. Pilon and T. Reiter, Comput. Phys. Commun. 180, 2317 (2009) [arXiv:0810.0992]. G. Cullen, J.P. Guillet, G. Heinrich, T. Kleinschmidt, E. Pilon, T. Reiter and M. Rodgers, Comput. Phys. Commun. 182, 2276 (2011) [arXiv:1101.5595].

[31] A. van Hameren, Comput. Phys. Commun. 182, 2427 (2011) [arXiv:1007.4716].

[32] F. Maltoni and T. Stelzer, JHEP 0302, 027 (2003) [hep-ph/0208156].

[33] M. Cacciari, G. P. Salam and G. Soyez, JHEP 0804, 063 (2008) [arXiv:0802.1189].

[34] M. Cacciari and G. P. Salam, Phys. Lett. B 641, 57 (2006) [hep-ph/0512210]. M. Cacciari, G. P. Salam and G. Soyez, Eur. Phys. J. C 72, 1896 (2012) [arXiv:1111.6097].

[35] H. -L. Lai et al., Phys. Rev. D 82, 074024 (2010) [arXiv:1007.2241].

[36] M.R. Whalley, D. Bourilkov and R.C. Group, hep-ph/0508110.

[37] T. Plehn, D. L. Rainwater and D. Zeppenfeld, Phys. Rev. Lett. 88, 051801 (2002) [hep-ph/0105325]. 